\title{
El Roque de Los Muchachos site characteristics
}

\section{Analysis of atmospheric dust and aerosol extinction}

\author{
G. Lombardi ${ }^{1,2,3}$, V. Zitelli ${ }^{2}$, S. Ortolani ${ }^{4}$, M. Pedani ${ }^{5}$, and A. Ghedina ${ }^{5}$ \\ 1 Department of Astronomy, University of Bologna, via Ranzani 1, 40127 Bologna, Italy \\ e-mail: gianluca.lombardi@oabo.inaf.it \\ 2 INAF-Bologna Astronomical Observatory, via Ranzani 1, 40127 Bologna, Italy \\ European Southern Observatory, Casilla 19001, Santiago 19, Chile \\ 4 Department of Astronomy, University of Padova, vicolo dell'Osservatorio 2, 35122 Padova, Italy \\ 5 Fundación Galileo Galilei and Telescopio Nazionale Galileo, PO Box 565, 38700 Santa Cruz de La Palma, Tenerife, Spain \\ Received 28 July 2007 / Accepted 13 February 2008
}

\section{ABSTRACT}

\begin{abstract}
Context. It is known that the Canary Islands are normally affected by dominant winds flowing from north-northeast, that in some meteorological conditions, can transport sand from the Sahara desert at high altitude. The dust may affect the efficiency of the telescopes and decrease the transparency of the sky.

Aims. To maximize the scientific return of the telescopes located at the Observatorio del Roque de Los Muchachos (ORM), we present an analysis of the atmospheric dust content and its effects on astronomical observations. Than $B, V$ and $I$ dust aerosol astronomical extinction are derived.

Methods. Using a 5-year series database of data taken from a dust monitor located inside the Telescopio Nazionale Galileo (TNG) dome, we computed mean hourly and daily values of the dust content as measured with a four-channel dust monitor.

Results. We detected particles of $0.3,0.5,1.0$, and $5.0 \mu \mathrm{m}$. Furthermore, using a power law we derived the content of $10.0 \mu \mathrm{m}$ particles. We found a typical local dust concentration ranging from $3 \times 10^{6}$ particles per cubic metre at $0.3 \mu \mathrm{m}$, to $10^{3}$ at $5.0 \mu \mathrm{m}$ and 10 at $10.0 \mu \mathrm{m}$, increasing up to 3 orders of magnitudes during the dust storms, with a relatively higher increase of $1.0,5.0$, and $10.0 \mu \mathrm{m}$ particles. The number of local dust storm events is the same in the local winter and summer, but the average background and storm-related increases in the dust concentration in summer are significantly higher than in winter. In a uniform approximation, during the dust storms, an average height of the dust layer of $2.5 \mathrm{~km}$ above the telescope is inferred.

Conclusions. During the sand storms, La Palma Island is affected by an almost uniform layer extending up to $5 \mathrm{~km}$ above the sea level. The visible extinction is dominated by particles at 1.0,5.0 and $10.0 \mu \mathrm{m}$. In agreement with the results from Carlsberg Automatic Meridian Circle (CAMC), we find a typical extinction of about 0.2 mag airmass $^{-1}$ during dust storms.
\end{abstract}

Key words. site testing - atmospheric effects - Earth

\section{Introduction}

The Telescopio Nazionale Galileo (TNG) is one of the international telescopes located at the Observatorio del Roque de Los Muchachos (ORM) on La Palma Island (Canary Arcipelago). Due to the proximity of the North African continent, the winds coming from the central side of the Sahara desert carry a large amount of dust in suspension that can reach high altitudes and increase the atmospheric dust concentration.

Whittet et al. (1987) pointed that this wind action removes approximately $2 \times 10^{8}$ tonnes of dust every year from Sahara desert, and they can be carried out to altitudes up to $6 \mathrm{~km}$. This seasonal action can reach the south of the Canary Islands, and extended dust clouds often appear above the sky of La Palma, originating a phenomenon locally named la Calima.

This natural event is often discuss concerning the quality of astronomical observations obtained at ORM such when quantify the contribution on astronomical extinction. Therefore a knowledge of the amount of the dust and, if possible, of the resulting extinction is very important for optimizing the performance of both instruments and telescopes and for obtaining reliable photometric measurements. Moreover, the knowledge of the grain sizes and their distribution at the mirror level is fundamental because the spectral index of the extinction depend on the size of grains. In fact, grains having dimensions larger than the adopted wavelength produce a neutral extinction, while sub-micron particles produce an extinction in the same band, with respect to the adopted wavelength.

The first detailed study of the impact of Calima on astronomical observations in La Palma is in Murdin (1985), who found a seasonal trend in atmospheric transmission, ranging from $90 \%$ of nights classified as good (i.e., nearly aerosol-free), between October and March, to 58\% during July, August, and September. Another interesting result from Murdin is the atmospheric extinction measured in a site-test campaign in La Palma. A Murdin summarisation of studies from other authors shows that $78 \%$ of the clear nights in the months starting from February to September 1975 and $83 \%$ from December 1974 to November 1975 have extinction less than 0.3 mag airmass $^{-1}$.

To improve the TNG meteorological station, the TNG site group put in operation a dust monitor. The first preliminary results can be found in Porceddu et al. (2002) and Ghedina et al. (2004). The primary motivation behind this measurement is to have a continuous characterisation of the air conditions above the primary mirror for several reasons. The first one is to 
compare dust measurements and atmospheric extinction to know which level of dust may affect astronomical observations. The second is to have better maintenance. In fact we know that the deposition of the dust over the optical surfaces reduces both the optical throughput (laboratory measurements of the primary mirror reflectivity show a decrease from $99 \%$ reached in the case of fresh aluminum to $70 \%$ in the case of a dusty surface) and the signal-to-noise ratio of the images. The third one is for safety reasons and for coating lifetimes, when humidity condensation sticks the dust on the mirror. The knowledge of the level of dust counts helps us to prevent damage to the optical surfaces of the telescope. Finally, the knowledge of the dust concentration at different sizes is also important for the proper design of the sealing of the telescopes and instruments moving mechanical parts.

The first detailed analysis of more than 10 years of meteorological data obtained using the TNG meteorological station at ORM can be found in the two recent papers, Lombardi et al. (2006, hereafter Paper I) and Lombardi et al. (2007, hereafter Paper II). Paper I shows a complete analysis of the vertical temperature gradients and their correlation with the astronomical seeing. Instead, Paper II shows an analysis of the correlation between wind and astronomical parameters, as well as the overall long-term weather conditions at ORM. Differences in the ORM microclimate demonstrated in a detailed comparison between synoptical parameters taken at three different locations in the observatory on a $1000 \mathrm{~m}$ spatial scale. Furthermore, ORM was shown to almost be dominated by high pressure and characterised by an averaged relative humidity lower than $50 \%$.

Extended dust clouds periodically appear above the ORM complex. Murdin (1985) shows that dust collected at the ground level at ORM is composed of small round quartz aggregates having diameters ranging between 10 to $60 \mu$ m diameter. The optical properties of this dust have been investigated by several authors (Stickland 1987; Murdin 1985; Jiménez et al. 1998), all founding a flat wavelength dependence of the extinction; in particular, Murdin points out that Saharian dust's size is much bigger than the standard photometric $V$ band wavelength.

Big plumes of dust during the Calima days are also visible using satellites probes. In Siher et al. (2004) an analysis of 5200 data covering the years 1978-1993 shows a correlation between astronomical extinction and the Total Ozone Mapping Spectrometer (TOMS) satellite derived extinction as well as a seasonal trend above the Canaries with a significant increase in dusty days at the end of July. Siher et al. (2004) also shows an average of 55 dusty days from the beginning of June to the beginning of September (about half of the summer nights). In this paper only the aerosol data corresponding to a significant absorption were used. The data since 1978 seems to indicate that the dusty period is drifting during the years. An independent analysis, based on Carlsberg Automatic Meridian Circle (CAMC) telescope data, has been published by Guerrero et al. (1998).

The present paper has the goal of improving the knowledge of the aerosol-particle-size distribution and the effects on astronomical observations in La Palma. In particular, we have analysed for the first time the influence of the dust collected at the level of TNG primary mirror (M1) as a function of its size to the aim: a) to understand if local measurements may be taken as good indicators of the upper conditions in the atmosphere (a dust ground condition has reproduced a similar situation in the atmosphere?); b) to give an accurate extinction study selecting among different sizes of dust; c) to use this information as a primary tool for a fast warning of the unclean environment.

The paper is organised as follows. Section 2 describes of the adopted particles counter. Section 3 describes the adopted procedures to analyse the collected data. Section 4 defines and reports number of storm events. A comparison between local extinction and total visual extinction obtained from the database of the CAMC is discussed in Sect. 5.

Using TOMS satellite archive, we have extracted the Aerosol Index from TOMS/EarthProbe, which provides independent, semi-quantitative indications of the dust content in the high atmosphere. Discussions of aerosol and TOMS data are in Sect. 6, conclusions can be found in Sect. 7.

\section{The dust monitor}

The selection of air-particles counting equipment is primary triggered by the particle sizes to be monitored to obtain their estimated amount. The parameters used for this selection were the sizes of the dust, the flow rate, and the background noise counts. Each measured size of the dust corresponds to the number of available channels of the counter. The sensitivity of the detector is given by the smallest size of particle that the sensor is able to detect. The flow rate is the amount of the flux that the sensor can receive through the sample volume, while the background noise is the instrument dark current value. We have addressed our choice to an easy-to-use instrument, hand-held, reliable, and very sensitive. Moreover, a particle counter based on the optical detection technique that can count particles above $5.0 \mu \mathrm{m}$ diameter was selected. The reason for such sensitivity is that we expect the number of dust particles to be less than the total number of atmospheric particles and we expect them to occur mostly on scales near $1 \mu \mathrm{m}$ with a typical concentration range of a few tens per cubic centimetre. We therefore chose the particle counter Abacus TM301 (made by Particle Measuring System, Inc.), which uses a laser scattering technique for environmental ambient air analysis. Abacus TM301 is a compact and portable system designed to measure the purity of close environments, like a clean room, the counter is a system to measures the density of particles in open air. This system is based on a filter through which the air is forced to flow. Different kinds of filters are used sequentially to measure different sizes of particles centered at $0.3,0.5,1.0$, and $5.0 \mu \mathrm{m}$ according to the estimated dimension of dust and sand of the Sahara desert as described by Murdin (1985). A small pump placed inside the dome sucks the air and, through scattering the light from a laser diode, measures the number of particles. The four sizes of particles can be measured in both integrated and differential modes. Although the counter is mounted inside the TNG dome and at the level of telescope, a $1 \mathrm{~m}$ long silicon pipe through the wall feeds the pump to external air at $13 \mathrm{~m}$ above the ground. This is a needed precaution because, even if we intend to use the counter to monitor the external air, it must be kept in a closed site, safe from adverse weather conditions. Unfortunately, we cannot distinguish between the type of particles, but only their size. For instance, it is not possible to distinguish between water vapour and dust. For this reason it is necessary to stop the monitoring if relative humidity increases to the condensation point (typically $\geq 85 \%$ ). Table 1 summarises the instrumental basic performances already reported in Porceddu et al. (2002) and also reported here for completeness.

\section{Data analysis}

The dust monitor measures outdoor dust at the same level as to TNG M1 to have a monitoring as close as possible the M1 local conditions. Data coming from Abacus TM301 are considered 


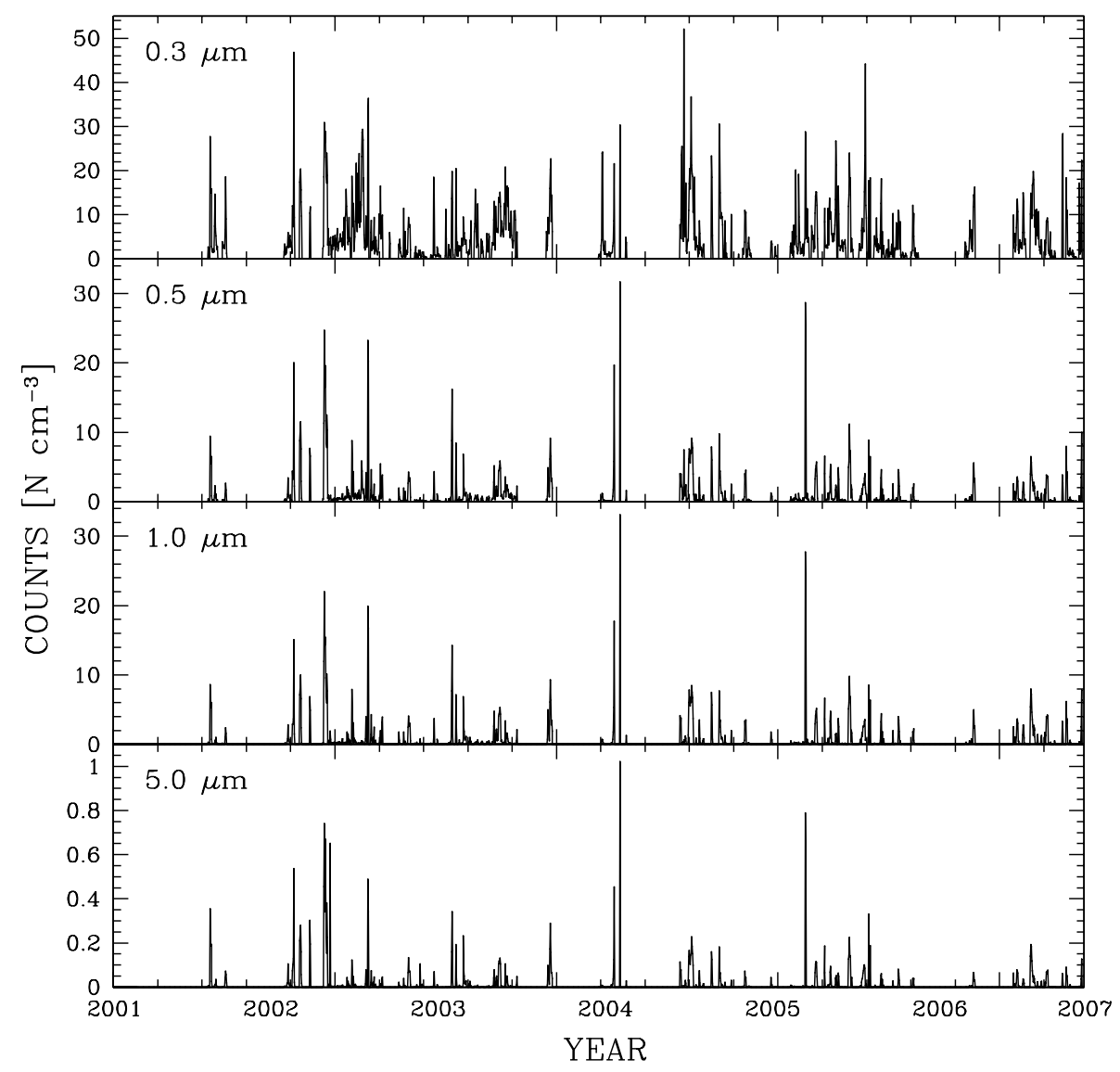

Fig. 1. Distribution of the atmospheric particles as a function of the different size, as measured by the Abacus TM301 during the years 2001-2006 (1-2 weeks each month depending on weather conditions). The gaps correspond to interruptions in the counter operations or to rejected values due to relative humidity $>85 \%$.

Table 1. Main characteristics of the Abacus TM301 dust monitor.

\begin{aligned} & \hline \hline size sensitivity $0.3 \mu \mathrm{m}$ at $50 \%$ counting efficiency \\ & input flow rate 0.1 Cubic Foot Minute [CFM] \\ & size channels $0.3,0.5,1.0,5.0 \mu \mathrm{m} \\ &$ light source laser diode $(\lambda=780 \mathrm{~nm}) \\ &$ concentration limits $\geq 10^{6}$ per dm instantaneous \\ & zero counts $<1$ per CF \\ & output RS-232 \\ & data storage 500 sample, rotating buffer \\ & sample time selectable from 1 s to 99 min \\ & purge time selectable from 1 s to $99 \mathrm{~min} \\ &$ dalay time selectable from 1 s to $99 \mathrm{~min} \\ &$\hline\end{aligned}

not as instantaneous values but as mean values after an integration time of $10 \mathrm{~min}$. Before each measurement, a 5-min long reset is used to clean the sensor. Abacus TM301 stores about 500 sets of data in memory, and when the memory is full, it uses an RS232 serial common port to transfer data to the PC for further analysis. Up to now the database collects measurements from August 2001 to December 2006, and it is used consecutively for 1-2 weeks each month depending to the weather conditions. In particular the counting is stopped if relative humidity $>85 \%$. In Figure 1 we report the full dust-count distribution for each particle size as measured during the years. The gaps in the figure corresponding to zero counts are caused by interruptions in the counter operations or by rejected values due to relative humidity $>85 \%$.
To check the presence of a seasonal variation, we split the dust database in two epochs, defining wintertime the months from October until March and summertime the months from April until September. In this analysis we define the dust storm event by each dust count having a value a few $\sigma$-levels over the monthly mean values. The background is evaluated using the $\sigma$-clipping algorithm also described in Huber (1981) and Patat (2003).

The particle counts $\left(N_{i}\right)$ were assumed to have a Poissonian distribution. For each month we computed the median value of the counts $(M E D)$, then for each different size of dust we calculated the median absolute deviation $(M A D)$ defined as the median of the distribution

$\left|N_{i}-M E D\right|$.

We set the $\sigma$ parameter to the value $\sigma=1.48 M A D$ (according to Huber 1981), where 1.48 is the ratio between the standard deviation and the MAD under the assumption of a Gaussian distribution. During the iteration we rejected counts having

$\left|N_{i}-M E D\right|>K \sigma$.

The procedure for estimating the dust background was iterated twice. In the first iteration we rejected the points exceeding $\pm 3 \sigma$ with respect to the computed monthly median. In the second iteration, we rejected points exceeding $2 \sigma$ and $-3 \sigma$ so that the background distribution is uncontaminated by any other dust peaks corresponding to dust storms.

We have found that, in connection with dust storms, counts of particles having diameters of $0.3,0.5$, and $1.0 \mu \mathrm{m}$ increase 


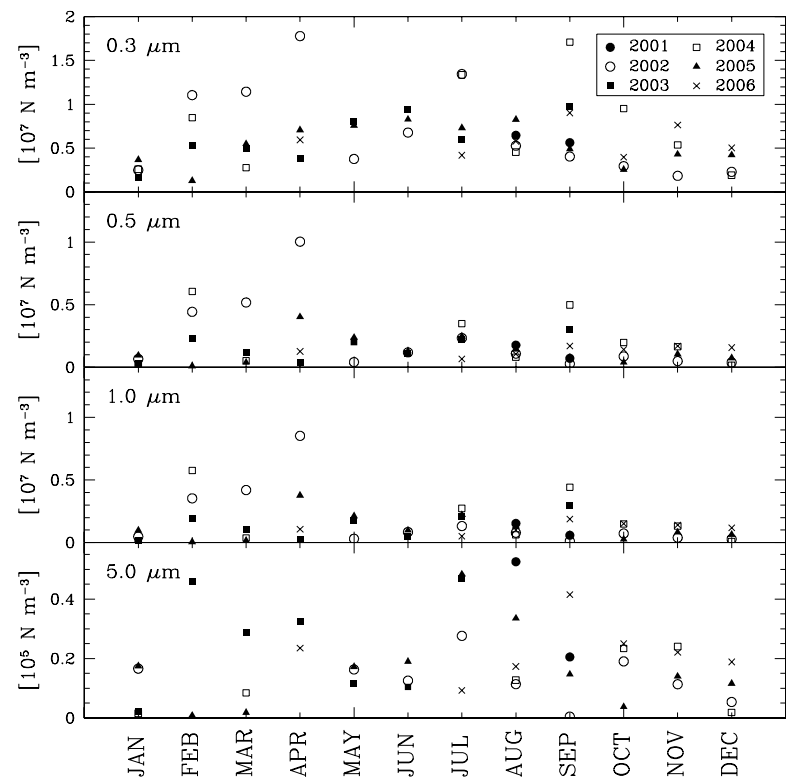

Fig. 2. Seasonal distribution of the monthly dust at ORM: (top) $0.3 \mu \mathrm{m}$ dust; (middle - top) $0.5 \mu \mathrm{m}$; (middle - bottom) $1.0 \mu \mathrm{m}$ dust; (bottom) $5.0 \mu \mathrm{m}$ dust. Different years are indicated by different symbols.

by 2 orders of magnitude with respect to the background. Unfortunately $5.0 \mu \mathrm{m}$ particles often present a typical fluctuation between 1 and 2 orders of magnitude, so it is difficult to distinguish dust storms from the typical dust background. For this reason an increase of 3 orders of magnitudes does allow us to distinguish the $5.0 \mu \mathrm{m}$ peaks from the background.

Figure 2 shows the median monthly distribution of the particles as a function of the month. It is evident that the level of background depends on size. The distribution shows the increase in the counts during February-April and July-September, while May and June are the months with the lower level of dust.

Table 2 shows the median dust content as computed in the two seasons and in the entire annual cycle. The table clearly shows that small particles are dominant both in wintertime and in summertime. It is not surprising because heaviest particles decay quickly, while lighter particles flow in the atmosphere for longer.

\section{Dust storms}

As mentioned, dust storm is the event where the content of dust, given in counts per cubic metre $\left[\mathrm{N} \mathrm{m}^{-3}\right]$, increases with respect to the typical dust background content in clear days by 2 orders of magnitude or more for $0.3,0.5$, and $1.0 \mu \mathrm{m}$ dust, and 3 orders of magnitude or more for $5.0 \mu \mathrm{m}$ dust. Figure 3 shows a more detailed situation of a dust storm that occurred in December 2002. This event persisted for two days, on 2002 December 25 and 26, and it shows an increase of several orders of magnitude in the counts.

In a deeper analysis we noticed that the dust storms are typically 3-4 days long, but sometimes it is possible to have some short storms 2 days long or, more rarely, long-lasting storms persisting 5-6 days. We correlated the wind direction with the duststorm counts. Figure 5 shows the wind roses obtained during the storm events as a function of particle size. The wind direction is recorded by the TNG meteorological tower and data were already analysed in Paper II. There is a clear correlation between dust storms and wind direction. Only the winds blowing from
Table 2. Dust background content in $\left[\mathrm{N} \mathrm{m}^{-3}\right]$ at ORM in wintertime, summertime, and in the entire annual cycle.

\begin{tabular}{lccc}
\hline \hline & Wintertime & Summertime & Annual \\
\hline $0.3 \mu \mathrm{m}$ & $1.27 \times 10^{6}$ & $4.38 \times 10^{6}$ & $2.98 \times 10^{6}$ \\
$0.5 \mu \mathrm{m}$ & $1.21 \times 10^{5}$ & $3.76 \times 10^{5}$ & $2.53 \times 10^{5}$ \\
$1.0 \mu \mathrm{m}$ & $0.51 \times 10^{5}$ & $1.51 \times 10^{5}$ & $1.02 \times 10^{5}$ \\
$5.0 \mu \mathrm{m}$ & $0.66 \times 10^{3}$ & $1.47 \times 10^{3}$ & $1.09 \times 10^{3}$ \\
\hline
\end{tabular}

Table 3. Number of storm events at ORM in wintertime, summertime, and in the entire annual cycle in the period from August 2001 to December 2006.

\begin{tabular}{lccc}
\hline \hline & Wintertime & Summertime & Total \\
\hline $0.3 \mu \mathrm{m}$ & 10 & 8 & 18 \\
$0.5 \mu \mathrm{m}$ & 15 & 12 & 27 \\
$1.0 \mu \mathrm{m}$ & 21 & 22 & 43 \\
$5.0 \mu \mathrm{m}$ & 9 & 7 & 16 \\
\hline
\end{tabular}

south-west, i.e. from the edge of the Caldera de Taburiente, carry a large amount of dust particles. We confirm that the best photometric conditions occur when the wind blows from the north.

In Table 3 the total number of events for both wintertime and summertime in the period from August 2001 to December 2006 is reported. Particles having 1.0 and $0.5 \mu \mathrm{m}$ size show the larger number of events. Table 3 shows that dust storms occur with the same statistic in both wintertime and summertime. Whittet et al. (1987) demonstrated that $95 \%$ of the aeolian deposits collected at La Palma have diameters between 1 and $100 \mu \mathrm{m}$. To better understand this analysis we calculated the median value of the dust content in case of storms for each measured size, and to include more massive grains we extrapolated the counts to $10.0 \mu \mathrm{m}$. Following Whittet et al. (1987), we assume a powerlaw distribution $r^{2} N(r)$ for particles $\geq 1.0 \mu \mathrm{m}$. Because we cannot distinguish between seasons, we decided to consider this background as a constant in both wintertime and summertime. Under these assumptions we found that $10.0 \mu \mathrm{m}$ particles have a typical background of $\sim 10 \mathrm{~N} \mathrm{~m}^{-3}$ which is a lower concentration than the other particles we measured. The same power law permits us to extrapolate the concentration to the $10.0 \mu \mathrm{m}$ size in case of dust storms. We obtained a concentration of $\sim 4 \times 10^{4} \mathrm{~N} \mathrm{~m}^{-3}$. Results of this analysis are plotted in Fig. 4.

\section{Aerosol atmospheric extinction in $B, V$, and $I$}

The optical properties of the dust in La Palma have been investigated by several authors who found that aerosol extinction does not have a clear dependence on the adopted wavelength. In particular, Stickland (1987) pointed out that extinction during dust storms is relatively stable on a time scale of $15 \mathrm{~min}$. No analysis exists for the effects of the different dust sizes on astronomical extinction.

In this paper we want to compare the differential extinction computed using our measured dust with simultaneous nighttime astronomical extinction obtained from the CAMC database, which is a very useful tool for quantitatively studying dust effects. We evaluate aerosol atmospheric extinction $(k)$ for each size of dust at 3 different wavelengths: 435,550 , and $780 \mathrm{~nm}$ corresponding to the central wavelengths of the standard photometric bands $B, V$, and $I$. We believe that using the differential dust counter may help us evaluate the different extinction contributions from the different dimensions of the grains as a function of the wavelength. 


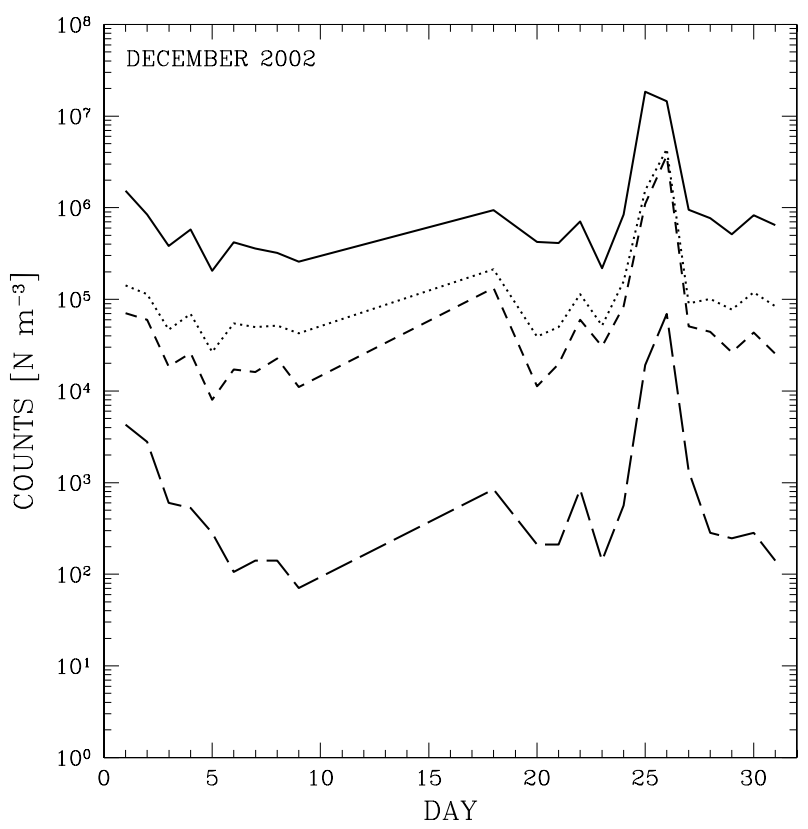

Fig. 3. Dust storm event of 2002 December 25 and 26: $0.3 \mu \mathrm{m}$ (solid), $0.5 \mu \mathrm{m}$ (dots), $1.0 \mu \mathrm{m}$ (short-dashes) and $5.0 \mu \mathrm{m}$ (long-dashes).

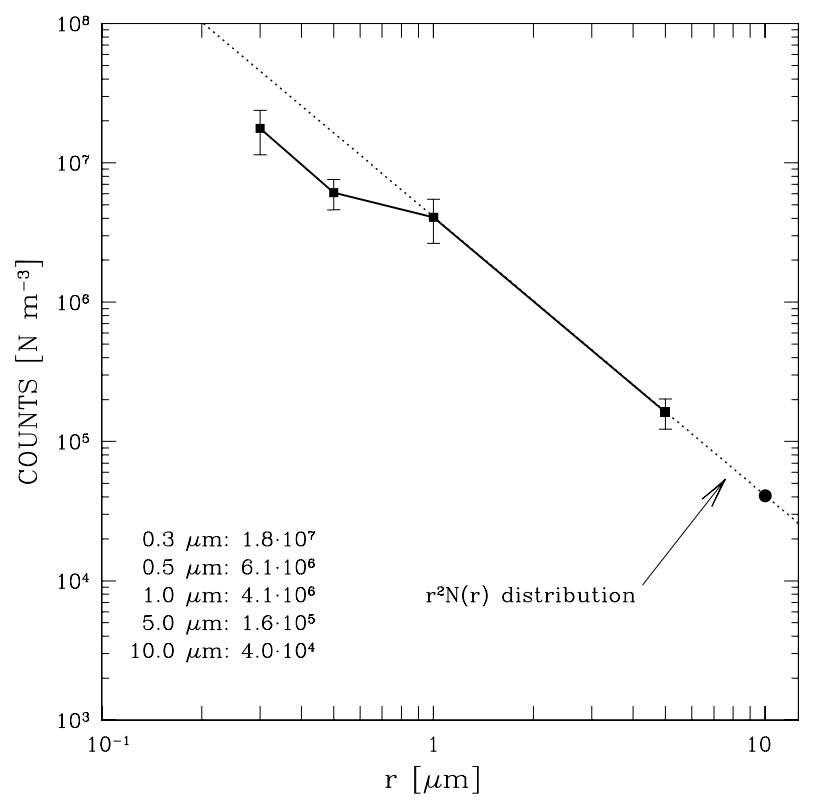

Fig. 4. Distribution of the median dust counts during dust storms. Particles $\geq 1.0 \mu \mathrm{m}$ are treated as if they follow an $r^{2} N(r)$ power law.

The aerosol extinction is obtained by applying the model based on the Mie theory and has also been used by Mathis et al. (1977), Whittet et al. (1987), Jiménez et al. (1998), and Marley et al. (1999). The Mie theory assumes the particles are homogeneous spheres of radius $r$. Following Patterson (1977) definitions, the scattering coefficients for a Mie solution are linked to the size and composition of the particles through the parameter $x=2 \pi r / \lambda$. For an incident light at wavelength $\lambda$, the extinction coefficient $k$ due to particles of radius $r$ is defined by the formula

$k_{\lambda, r}=Q_{\mathrm{ext}}(n, r, \lambda) \pi r^{2} N$

where the term $Q_{\text {ext }}(n, r, \lambda)$ is the extinction efficiency factor computed for Mie's particles, while $N$ is the number of particles of radius $r$ in a cubic centimetre. With this definition, $k_{\lambda, r}$ is

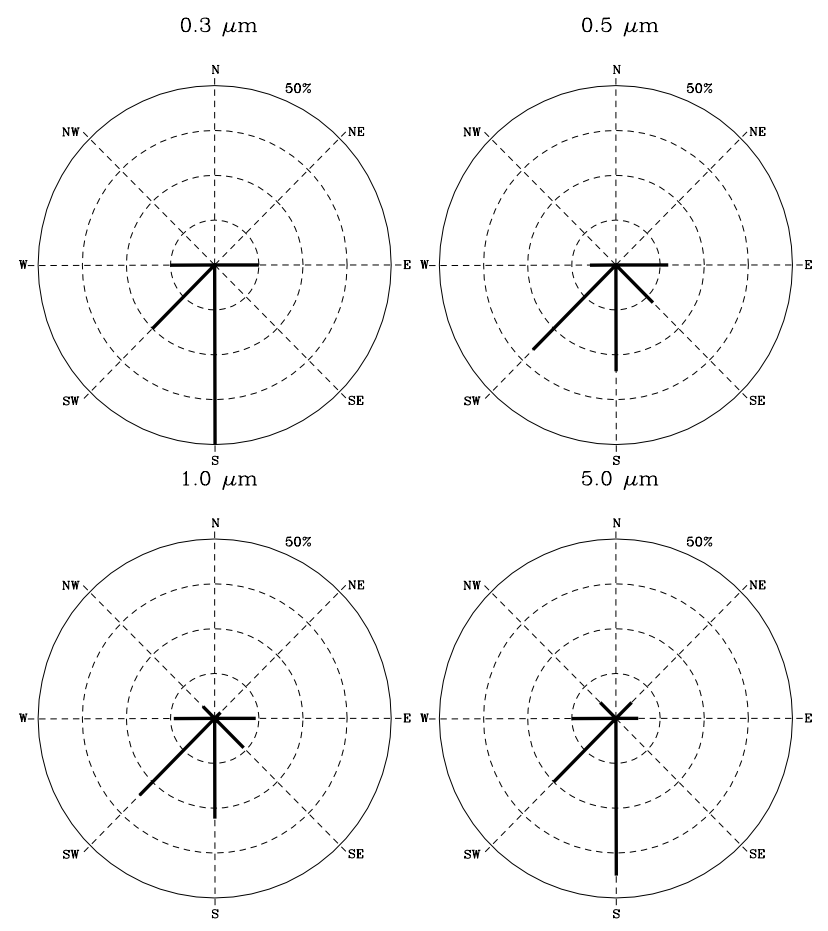

Fig. 5. Wind roses at TNG during dust storm events.

Table 4. Values of the extinction efficiency factor $Q_{\text {ext }}$.

\begin{tabular}{rccc}
\hline \hline & $B$ & $V$ & $I$ \\
\hline $0.3 \mu \mathrm{m}$ & 4.148 & 4.334 & 2.783 \\
$0.5 \mu \mathrm{m}$ & 1.946 & 2.756 & 4.137 \\
$1.0 \mu \mathrm{m}$ & 2.367 & 2.355 & 2.205 \\
$5.0 \mu \mathrm{m}$ & 2.142 & 2.135 & 2.140 \\
$10.0 \mu \mathrm{m}$ & 2.072 & 2.084 & 2.099 \\
\hline
\end{tabular}

expressed in magnitudes per centimetre $\left[\mathrm{mag} \mathrm{cm}^{-1}\right]$ and is correlated with the local density of the dust content in the atmosphere above the TNG dome.

In the equation, the extinction efficiency factor $Q_{\text {ext }}$ is a complex function depending on the particles radius $r$, the absorbed wavelength $\lambda$ and the complex refractive index $n$. In the definition (Patterson 1981), the real part of $n$ is the ratio between light speed in a vacuum and the light speed in particles, while the imaginary part is related to the Bouguer absorption coefficient $\kappa$ through the formula $n_{\mathrm{im}}=\kappa \lambda / 4 \pi$. Following Patterson (1977); Carlson \& Benjamin (1980); Fouquart et al. (1987), and Jiménez et al. (1998), we assigned $n=1.55-0.005 \mathrm{i}(\mathrm{i}=\sqrt{-1})$ as the typical refractive index for Saharan dust aerosols.

We computed the extinction efficiency factor $Q_{\text {ext }}$ for the adopted photometric bands using the Mie scattering code of Wiscombe (1980). Figure 6 shows the plot of $Q_{\text {ext }}$ as a function of the particle radius for $B, V$, and $I$ bands, while in Table 4 we report the computed values of $Q_{\text {ext }}$ for $0.3,0.5,1.0$, and $5.0 \mu \mathrm{m}$ dust in the three bands. We show that for $r \geq 1.0 \mu \mathrm{m}, Q_{\text {ext }}$ is almost constant in the three bands, confirming that for massive grains the extinction is colour-neutral as pointed out by Jiménez et al. (1998).

Applying Eq. (3), we obtained the local aerosol atmospheric extinction in [mag $\mathrm{cm}^{-1}$ ] caused by different grain sizes in typical dust background conditions $\left(k_{\mathrm{MED}}\right)$ and in the presence of dust storm events $\left(k_{\text {dusty }}\right)$.

Table 5 reports the $k_{\mathrm{MED}}$ in $B, V$, and $I$ in wintertime, summertime, and in the entire annual cycle using data as reported 


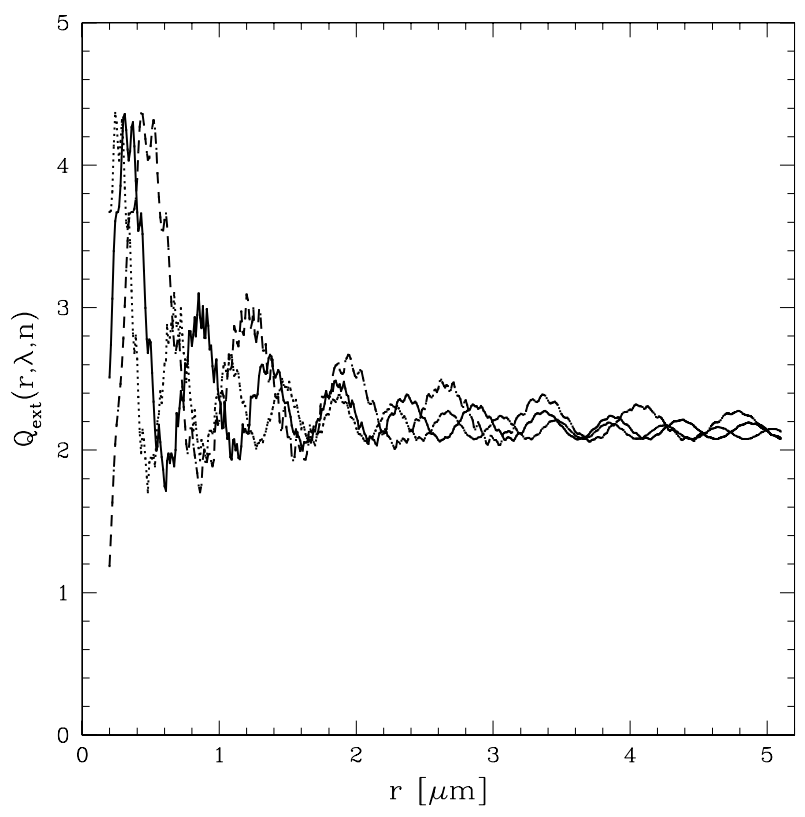

Fig. 6. Extinction efficiency factor as a function of the particles radius in $B$ (dots), $V$ (solid), and $I$ (short-dashes).

Table 5. Seasonal and annual local aerosol atmospheric extinction computed in dusty background conditions ${ }^{a}$.

\begin{tabular}{cccc}
\hline \hline & \multicolumn{3}{c}{ Wintertime } \\
& $k_{\mathrm{MED}}(B)$ & $k_{\mathrm{MED}}(V)$ & $k_{\mathrm{MED}}(I)$ \\
\hline $0.3 \mu \mathrm{m}$ & 1.49 & 1.56 & 0.99 \\
$0.5 \mu \mathrm{m}$ & 0.19 & 0.26 & 0.39 \\
$1.0 \mu \mathrm{m}$ & 0.38 & 0.38 & 0.35 \\
$5.0 \mu \mathrm{m}$ & 0.11 & 0.11 & 0.11 \\
$10.0 \mu \mathrm{m}$ & $<0.01$ & $<0.01$ & $<0.01$ \\
\hline \multicolumn{4}{c}{ Summertime } \\
& $k_{\mathrm{MED}}(B)$ & $k_{\mathrm{MED}}(V)$ & $k_{\mathrm{MED}}(I)$ \\
\hline $0.3 \mu \mathrm{m}$ & 5.14 & 5.37 & 3.45 \\
$0.5 \mu \mathrm{m}$ & 0.58 & 0.81 & 1.22 \\
$1.0 \mu \mathrm{m}$ & 1.12 & 1.12 & 1.05 \\
$5.0 \mu \mathrm{m}$ & 0.25 & 0.25 & 0.25 \\
$10.0 \mu \mathrm{m}$ & $<0.01$ & $<0.01$ & $<0.01$ \\
\hline \multicolumn{4}{c}{ Annual } \\
& $k_{\mathrm{MED}}(B)$ & $k_{\mathrm{MED}}(V)$ & $k_{\mathrm{MED}}(I)$ \\
\hline $0.3 \mu \mathrm{m}$ & 3.49 & 3.65 & 2.34 \\
$0.5 \mu \mathrm{m}$ & 0.39 & 0.55 & 0.82 \\
$1.0 \mu \mathrm{m}$ & 0.76 & 0.76 & 0.71 \\
$5.0 \mu \mathrm{m}$ & 0.18 & 0.18 & 0.18 \\
$10.0 \mu \mathrm{m}$ & $<0.01$ & $<0.01$ & $<0.01$ \\
\hline
\end{tabular}

${ }^{a}$ Values are given in $\left[10^{-8} \mathrm{mag} \mathrm{cm}^{-1}\right]$.

in Table 2 and for the extrapolated value of dust background at $10.0 \mu \mathrm{m}$. As shown in the table, on clear days $0.3 \mu \mathrm{m}$ dust gives the highest contribution to the global extinction, in particular in summertime. The extinction due to $0.3 \mu \mathrm{m}$ dust is lower at higher wavelengths, while for $0.5 \mu \mathrm{m}$ dust it becomes stronger at higher wavelengths, in particular in the summertime. Finally, the effect of the grains starting from $5.0 \mu \mathrm{m}$ is almost constant in the three bands. We found that the contribution of the extinction due to $10.0 \mu \mathrm{m}$ particles is negligible.

To understand the aerosol contribution on the total astronomical extinction, we need to evaluate the distribution in altitude of the particles. We compare our local extinctions in $V$ to simultaneous astronomical extinctions in $V$ from the CAMC database

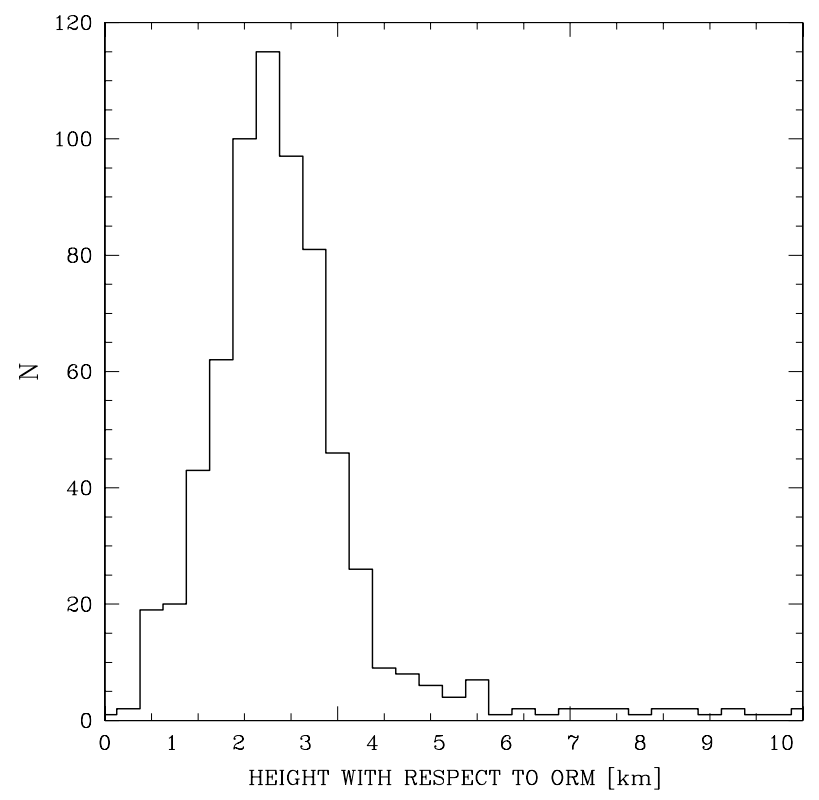

Fig. 7. Distribution of the dust altitudes from ORM altitude for Eq. (5).

$\left(k_{\mathrm{CAMC}}\right)$ under the assumption that $k_{\mathrm{CAMC}}$ is mainly due to our measured dust. We assume

$k_{\text {aer }}(\lambda)=k_{\lambda, 0.3}+k_{\lambda, 0.5}+k_{\lambda, 1.0}+k_{\lambda, 5.0}+k_{\lambda, 10.0}$.

Because the CAMC-extinction database is only in $V$ band, we compare our $V$ band aerosol extinctions. For each $k_{\text {aer }}(V)$ we assume that particles are distributed in the atmosphere at an altitude $h$, in order to have

$$
k_{\text {aer }}(V) h=k_{\text {CAMC }}(V) \text {. }
$$

We assume again that dust is uniformly distributed in altitude in a column of atmosphere having a base of $1 \mathrm{~cm}^{2}$ and height $h$ as obtained from Eq. (5). Figure 7 shows the distribution of the altitudes above the ORM obtained from each computed mean dust count. The maximum of the histogram shows the altitude reached by the dust. We found that the peak of the altitude distribution of the dust is about $2.5 \mathrm{~km}$ above the telescope. This value agrees very closely with studies based on independent radiosonde data (Hsu 1999) and indicates that the settlement process of the dust from the Sahara to La Palma, in the size range $0.3-10.0 \mu \mathrm{m}$, is not relevant, in agreement with the models of Murphy (1990). We conclude that, under the assumption of a uniform distribution of the dust, the particles are distributed in the first $2.5 \mathrm{~km}$, on average. We then recompute the aerosol atmospheric extinction using Eq. (3), integrating on a column of atmosphere of $2.5 \mathrm{~km}$. We assumed this obtained altitude to be equivalent to 1 airmass and the final extinction is therefore given in [mag airmass $\left.{ }^{-1}\right)$.

The $k_{\lambda, r}$ for each particle size in typical dust background conditions and in typical dust storm conditions are now recomputed by integrating on a $2.5 \mathrm{~km}$ column of atmosphere above the TNG mirror. Figure 8 shows the aerosol atmospheric extinction $k_{\lambda, r}$ in $\left[\mathrm{mag}\right.$ airmass $^{-1}$ ] and integrated on a column of atmosphere of $2.5 \mathrm{~km}$ in the entire annual cycle as a function of the size of the dust (cf. Table 2 and Fig. 4) for clear and dusty days.

We show that on clear days, the extinction is dominated by $0.3 \mu \mathrm{m}$ particles. Table 6 reports the percentage of contribution by different size of dust in each filter on both clear and dusty days. As shown in the table, on clear days $0.3 \mu \mathrm{m}$ particles are responsible for more than $70 \%$ of the total aerosol extinction in $B$ 


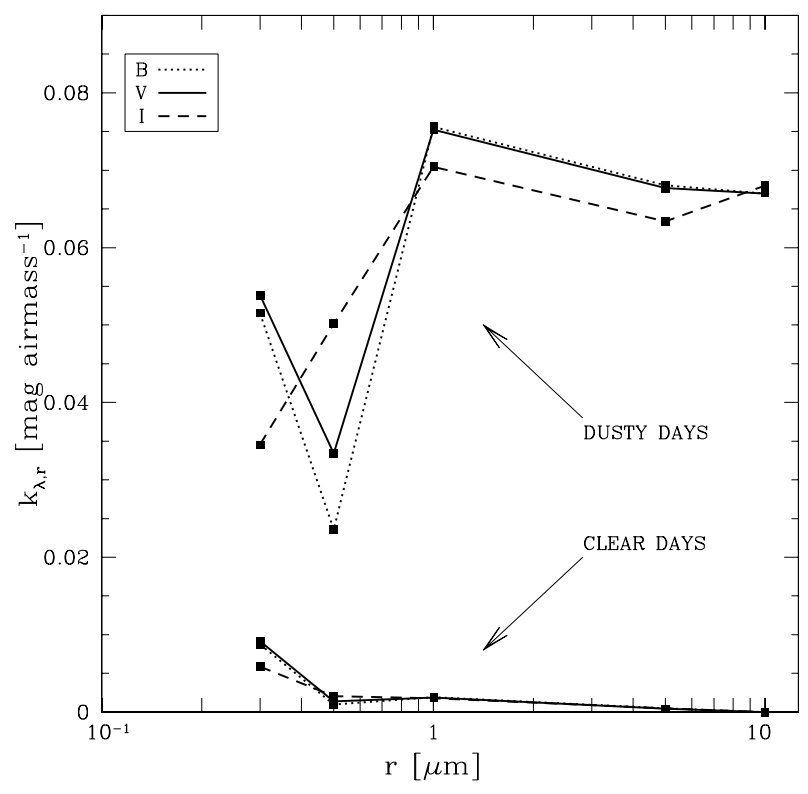

Fig. 8. Median aerosol atmospheric extinction in $B, V$, and $I$ in typical dust background conditions and in typical dust-storm conditions for each particle size. The biggest particles are dominant with respect to sub-micron particles.

Table 6. Relative contribution of each particle size as percentage of the total aerosol atmospheric extinction $k_{\text {aer }}$.

\begin{tabular}{cccccc}
\hline \hline \multicolumn{5}{c}{ Clear days } \\
& $0.3 \mu \mathrm{m}$ & $0.5 \mu \mathrm{m}$ & $1.0 \mu \mathrm{m}$ & $5.0 \mu \mathrm{m}$ & $10.0 \mu \mathrm{m}$ \\
\hline$B$ & 72 & 8 & 16 & 4 & $<0.1$ \\
$V$ & 71 & 11 & 14 & 4 & $<0.1$ \\
$I$ & 58 & 20 & 17 & 5 & $<0.1$ \\
\hline \multicolumn{5}{c}{ Dusty days } \\
\hline$B$ & $0.3 \mu \mathrm{m}$ & $0.5 \mu \mathrm{m}$ & $1.0 \mu \mathrm{m}$ & $5.0 \mu \mathrm{m}$ & $10.0 \mu \mathrm{m}$ \\
\hline$V$ & 18 & 8 & 26 & 24 & 24 \\
$I$ & 18 & 11 & 25 & 23 & 23 \\
\hline
\end{tabular}

and $V$, while their contribution in $I$ is about $60 \%$. The biggest particles $(\geq 1.0 \mu \mathrm{m})$ dominate in dusty days. It is interesting to note the different behaviors of $I$ band during dust storms with respect to the $B$ and $V$ bands. Bigger particles show a contribution to the total extinction of more than $70 \%$ in all the bands (see Table 6).

Following Eq. (4), the expected total local aerosol atmospheric extinction has to be calculated as the sum of the contributions of each particles size. On dusty days this extinction is typically $\sim 0.2$ mag airmass $^{-1}$ (see Fig. 8 ) and can rise to $\sim 1.0 \mathrm{mag}$ airmass $^{-1}$ during very strong dust storms (see Fig. 9). Figure 9 shows $k_{\text {aer }}(V)$ calculated on dusty days compared to simultaneous astronomical extinctions in $V$ band as extracted from the CAMC database. Figure 9 shows a good correlation, the dotted line is the linear fit at a confidence level of 0.8 and having a slope of 0.77 . We can conclude that it is possible to have an estimation of astronomical extinction, starting from a measure of dust count and by an appropriate calibration.

To evaluate the increase in the local aerosol extinction in connection with dust storm events, we also evaluated the differences between (i) the aerosol extinctions calculated on each day of a dust storm and (ii) the extinction calculated in the typical dust background conditions of each defined seasonal epoch:

$\Delta k(\lambda)=k_{\text {dusty }}(\lambda)-k_{\mathrm{MED}}(\lambda)$

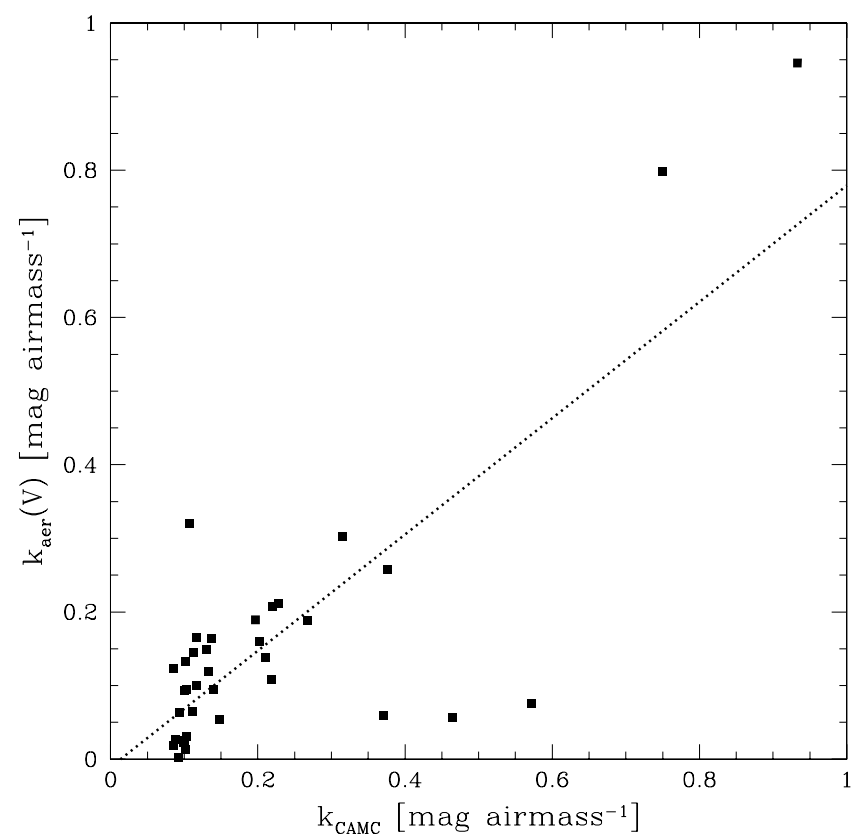

Fig. 9. $k_{\text {aer }}$ versus $k_{\text {CAMC }}$ in the case of dust storms. The linear fit has a confidence level of 0.8 .

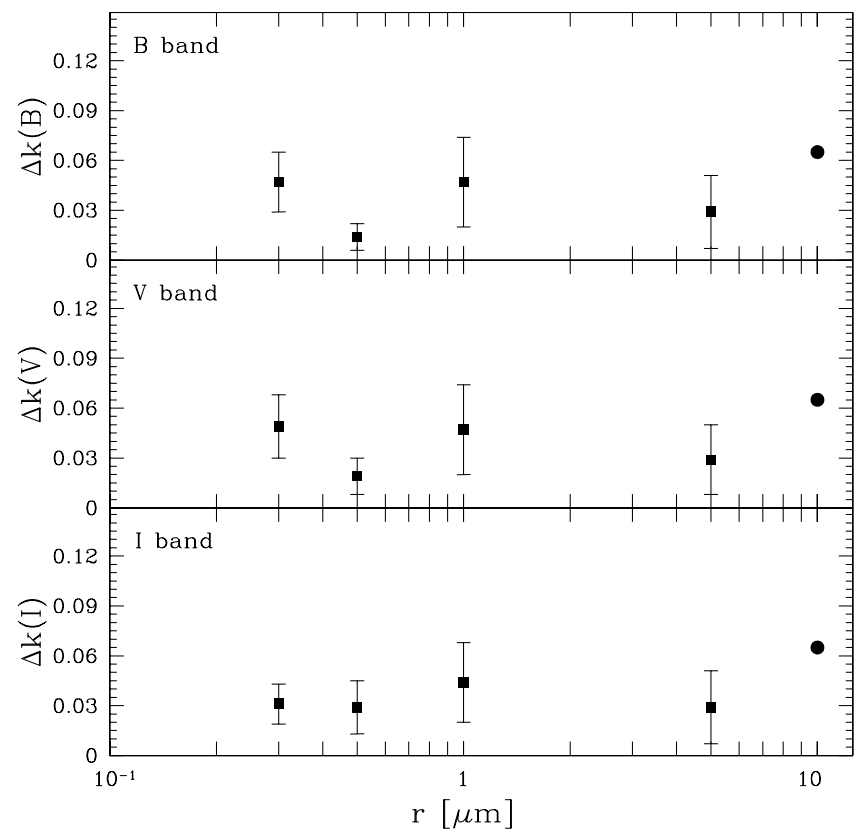

Fig. 10. Typical local aerosol extinction increases $\Delta k(\lambda)$ in [mag airmass ${ }^{-1}$ ] in $B$ (top), $V$ (center) and $I$ (bottom) in connection with dust storm events at ORM. The filled circles represent the expected $\Delta k(\lambda)$ due to $10 \mu \mathrm{m}$ particles.

Figure 10 plots the mean increases calculated for each dust size in $B, V$, and $I$. In the figure we have also taken the extrapolated extinction due to $10.0 \mu \mathrm{m}$ particles into account. The typical increases are between 0.015 and 0.065 mag airmass ${ }^{-1}$.

\section{Summertime aerosol atmospheric extinction and TOMS aerosol index}

Since from the extinction data alone it is not possible to distinguish cirrus clouds from dust clouds, we have combined both satellite and ground-based data comparing the daily $k_{\text {aer }}(\lambda)$ 
computed in summertime in the three photometric bands and the tropospheric TOMS aerosol index (AI) that characterises the local aerosol concentration using satellite-based reflectivity measurements. The TOMS data are set with the data closest to the ORM within a $1 \times 1 \mathrm{deg}$ latitude and longitude box. TOMS AI is selected on the same days as $k_{\text {aer }}(\lambda)$. Following Siher et al. (2004) we have set our threshold at AI $>0.7$. Because TOMS AI measurements above the Canaries are done in daytime, we obtained the nighttime AI by interpolating the values of two consecutive days. Satellite AI is obtained by the reflectivity effect. Because both dust and clouds may show reflectivity even at different threshold, we rejected AI points when sky reflectivity $>15 \%$ (Bertolin 2005) to be sure to have only the dust effect.

Once more we split the data between wintertime and summertime, and we noticed the absence of AI points in wintertime in connection with our local aerosol extinction calculations. Most of those points are the ones previously rejected for high sky reflectivity. In fact, in wintertime the sky is often contaminated by high reflectivity caused by thin cirrus or snow at the ground. Furthermore, in wintertime the dusts are often at low altitudes, too deep in the atmosphere to be revealed by the satellite.

Figure 11 shows the plot of the local aerosol atmospheric extinction in summertime versus the AI in $V$ band ( $B$ and $I$ show similar behaviours). In the plot we can distinguish three areas. The locus between the solid and short-dashed lines include all points having a correlation between $k_{\text {aer }}(\lambda)$ and AI with a confidence level $>0.8$ (Spearman's test). The points below the shortdashed line show high AI but low local extinction. This high reflectivity is caused by the Saharan dust suspended at a very high level that is not detectable at the TNG mirror level. The (very few) points above the solid line show high local extinction and low AI. We ascribe the cause to local, recycled dust that remain suspended at low altitude and cannot be revealed by the satellite.

To support this affirmation, we computed the two wind roses for the data subsets relative to those days on which we believe that the extinction is due to local recycled dust, and on those days when the high reflectivity comes from the Saharan dust suspended in the high atmosphere. We found that, in the case of local recycled dust, the winds come prevalently from W-SW, while in the case of dust suspended in the high atmosphere the prevailing direction is from $\mathrm{S}-\mathrm{SW}$.

We can conclude that observations may often be affected also by local recycled dust and not only by Calima events. More data may help us this point. Taking all the reasons mentioned above into account we conclude that in site testing, both local dust monitors and data from satellites have to be used together for a complete investigation of the effects of the aerosols.

\section{Conclusions}

The high-altitude transport events of dust from desert areas have been recognised the major atmospheric events on the Earth. The resulting pollution has astronomical observations and instrumentation (i.e. the main mirror reflectivity) effected even at very great distances, in particular at the Observatorio del Roque de Los Muchachos, on La Palma Island.

In this paper we have presented an analysis of the properties of the local atmospheric dust measured at the height of the TNG primary mirror, the correlations of the dust peak events with the wind direction, and the total atmospheric extinction using the 5-year long data archive of the TNG dust monitor. This particle counting system is able to detect particles of 0.3 , $0.5,1.0$, and $5.0 \mu \mathrm{m}$, thereby according with the estimated dimension of dust and sand of the Sahara desert. Typical annual

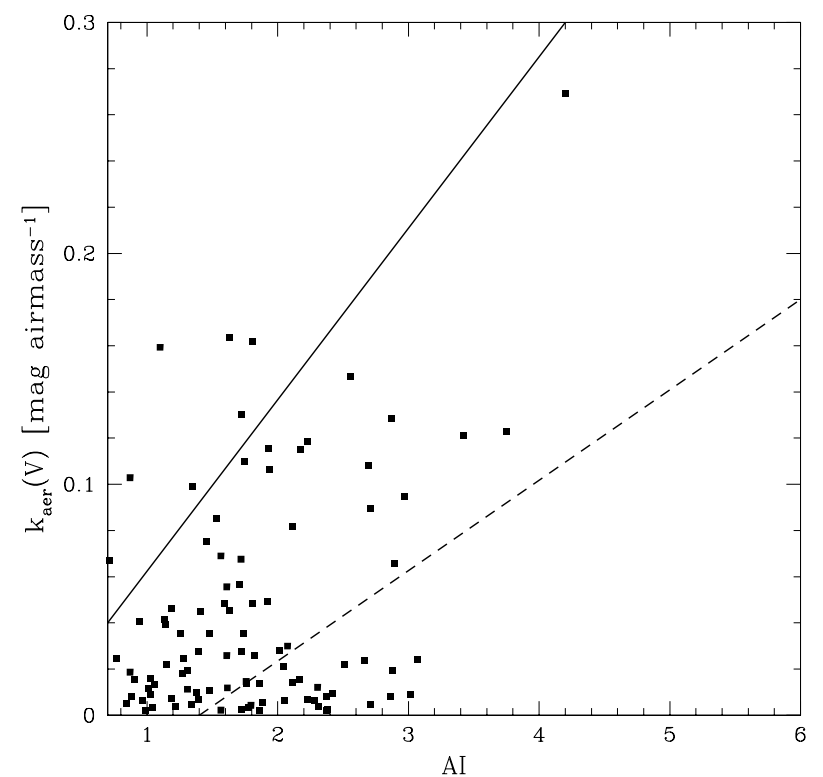

Fig. 11. Summertime local aerosol atmospheric extinction versus TOMS Aerosol Index in $V$ band. The correlation calculated with the Spearman's test between the points inside the zone delimited by the solid and the short-dashed lines has confidence level $>0.8$.

average background values are $3 \times 10^{6}, 2.5 \times 10^{5}, 10^{5}, 10^{3}$ particles per cubic metre, respectively, in the four channels. During dust storm events, the particle concentration increases to three orders of magnitude. Using a power-law distribution we also extrapolated the contents of $10.0 \mu \mathrm{m}$ dust on dusty days. We estimated an amount of $4 \times 10^{4}$ particles per cubic metre for massive grain.

After considering two seasonal epochs, we found the number of dusty events to be comparable, but they reach a somewhat higher concentration in summertime, when the background is also higher by a factor 3-4. In both seasons the concentration of small dust particles $(0.3 \mu \mathrm{m})$ is dominant with respect to the other size dust particles. A study of dust-storm events versus wind direction has shown that winds coming from the southwest, i.e. from the edge of the Caldera de Taburiente, typically carry a large amount of dust particles. Dust storms are 3-4 days long, but sometimes it is possible to have some shortly storms 2 days longer or, more rarely, long-lasting storms persisting 5-6 days.

A comparison between the storm duration and the TOMS Aerosol Index shows that short-storms typically are not linked with a high Aerosol Index. This suggests that short-storms can be generated by local reprocessing of currents carrying dust in suspension at low altitudes such is the TNG mirror level. We also noticed that dust storms occur not only in summertime (Saharan Calima events), but that several events occur during other seasons as effect of local currents recircle.

In clear days the aerosol extinction is dominated by $0.3 \mu \mathrm{m}$ particles $(\sim 70 \%)$, while in dusty days the strongest contribution is from bigger particles having size $\geq 1.0 \mu \mathrm{m}(>70 \%)$. On dusty days the extinction is typically $\sim 0.2$ mag airmass $^{-1}$, and it can rise to $\sim 1.0$ mag airmass $^{-1}$ in the presence of very strong dust storms.

In dusty days dust $V$ extinction shows a positive correlation with simultaneous astronomical extinctions values from CAMC database (c.l. 0.8). This suggests that the dust detected at TNG affects the astronomical observations in a very wide area.

Assuming a uniform distribution of the dust in the observing column, we found that the dust extends about $2.5 \mathrm{~km}$ above 
the telescope, corresponding to an height of $5 \mathrm{~km}$ above the see level. This confirms that the settlement process of the dust in the size range $0.3-10.0 \mu \mathrm{m}$, is not relevant.

Acknowledgements. The authors acknowledge the anonymous reviewer for the very useful comments and the TNG staff for the availability of the data. The authors thank also Guido Barbaro of Department of Astronomy of University of Padova for helpful comments on Mie's theory and Chiara Bertolin of Atmospheric and Climate Science Institute of National Research Council of Padova for the help in using TOMS/EarthProbe data.

\section{References}

Bertolin, C. 2005, private communication

Carlson, T. N., \& Benjamin, S. G. 1980, J. Atmos. Sci., 37, 193

Fouquart, Y., Bonnel, B., Chaoui Roquai, M., Santer, R., \& Cerf, A. 1987, J. Climate Appl. Meteor., 26, 28

Ghedina, A., Pedani, M., Guerra, J. C., Zitelli, V., \& Porceddu, I. 2004, SPIE, 5489, 227

Guerrero, M. A., García-López, R. J., Corradi, R. L. M., et al. 1998, New Astron. Rev., 42, 529
Jiménez, A., Gonzalez J. H., \& Rabello-Soares, M. C. 1998, A\&AS, 129, 413 Huber, P. J. 1981, Robust Statistics (New York: J. Wiley, \& Sons)

Hsu, N. C., Herman, J. R., Torres, O., et al. 1999, J. Geophys. Res., 104, 6269 Lombardi, G., Zitelli, V., Ortolani, S., \& Pedani, M. 2006, PASP, 118, 1198 (Paper I)

Lombardi, G., Zitelli, V., Ortolani, S., \& Pedani, M. 2007, PASP, 119, 292 (Paper II)

Marley, M. S., Gelino, C., Stephens, D., Lunine, J. I., \& Freedman, R. 1999, ApJ, 513,879

Mathis, J. S., Rumpl, W., \& Nordsieck, K. H. 1977, ApJ, 217, 425

Murdin, P. 1985, Vistas Astron., 28, 449

Murphy, J. R., Toon, O. B., Haberle, R. M., \& Pollack, J. B. 1990, J. Geophys. Res., 95, 14629

Patat, F. 2003, A\&A, 401, 797

Patterson, E. M. 1977, Appl. Opt., 16, 2414

Patterson, E. M. 1981, Science, 211, 836

Porceddu, I., Zitelli, V., Buffa, F., \& Ghedina, A. 2002, SPIE, 4844, 358

Siher, E. A., Ortolani, S., Sarazin, M., \& Benkhaldoun, Z. 2004, SPIE, 5489, 138 Stickland, D. J., Lloyd, C., Pike., C. D., \& Walker, E. N. 1987, The Observatory, 107, 74

Wiscombe, W. J. 1980, Appl. Opt., 19, 1505

Whittet, D. C. B., Bode, M. F., \& Murdin, P. 1987, Vistas Astron., 30, 135 